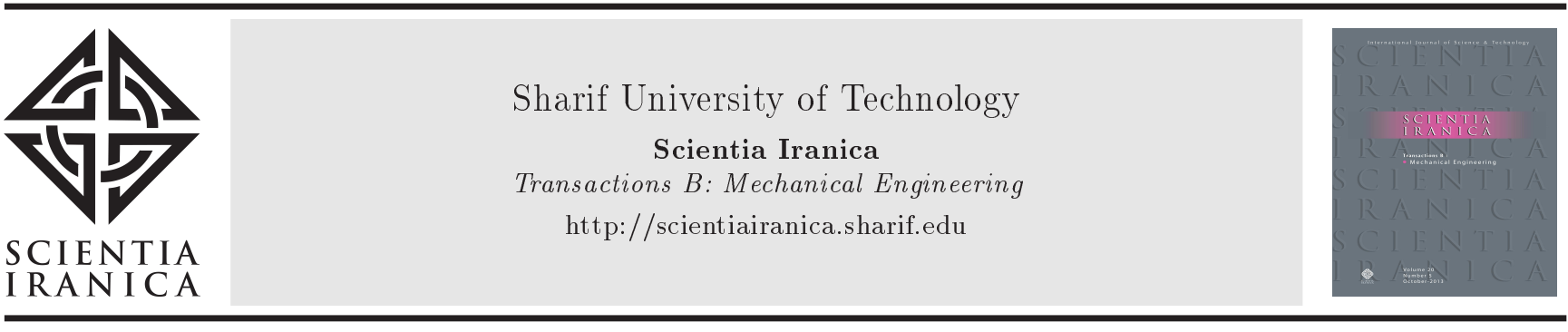

\title{
Development of a saturation-based $\mu(I)$-rheology for wet granular materials using discrete element method
}

\author{
R. Ghorbani, M.T. Manzari*, and A. Hajilouy-Benisi \\ School of Mechanical Engineering, Sharif University of Technology, Tehran, P.O. Box 11155/9567, Iran.
}

Received 16 October 2020; received in revised form 29 November 2020; accepted 8 February 2021

\section{KEYWORDS \\ Granular material; Rheological model; Discrete element method; \\ Inertia number; \\ Water saturation.}

\begin{abstract}
The present study employs Discrete Element Method (DEM) to establish a rheological model that relates the apparent viscosity of a granular material to shear rate, normal stress, and water saturation. In addition, a theoretical model was developed to determine water distribution and water-induced forces between particles for different saturations. The resulting forces were embedded in a 3D shear cell as a numerical rheometer, and a wet specimen was sheared between two walls. A power law rheological model was then obtained as a function of inertia number and saturation. It was found that up to a critical saturation, the apparent viscosity increased with saturation that was higher than that of the dry specimen. However, when the saturation exceeded the critical value, the viscosity suddenly dropped below that of the dry condition. To evaluate the model, the collapse of two-dimensional granular material on a horizontal rigid bed was studied using continuum-based numerical simulation which utilized the proposed rheological model.
\end{abstract}

(C) 2021 Sharif University of Technology. All rights reserved.

\section{Introduction}

A detailed study of the effect of water content on the rheological behavior of granular materials can provide a better understanding of the modeling of rainfallinduced landslides since according to the studies, water infiltration plays a critical role in the occurrence of landslides [1,2]. In fact, by establishing a relationship among the material viscosity, shear rate, normal stress, and water saturation through a continuum approach, a numerical method can be proposed to study the movement of wet granular materials.

During rainfall, water starts to infiltrate from the top and saturates the layers near the surface. As time passes, the wetting front moves downward and

*. Corresponding author. Tel.: +982166165689 E-mail address: mtmanzari@sharif.edu (M.T. Manzari)

doi: $10.24200 /$ sci. 2021.56993 .5014 the thickness of the saturated layer increases. In this saturated zone, pore water pressure is hydrostatic and positive which increases upon increasing the depth. In such layers, the inter-particle forces are affected by buoyancy which reduces the effective normal stress. Below the saturated front, due to the surface tension effect, water is trapped in the inter-particle spaces, thus creating an unsaturated zone. In the unsaturated layers, the pore pressure is negative and it pulls the particles together. Therefore, the normal stress and inter-particle forces change in the material depending on the saturation.

Different constitutive equations have been proposed to describe the dynamic behavior of granular materials. In this respect, the visco-plastic HerschelBulkley rheology [3] is widely utilized which establishes a non-linear relationship between the shear stress $\tau$ and shear rate $\dot{\gamma}$, which in turn uses the yield stress $\tau_{y}$ [4]. In this model, the material will be deformed if the applied shear stress exceeds the yield stress obtained 
from the Mohr-Coulomb failure criterion [5]. The $\mu(I)$ rheology [6] which defines the apparent viscosity $(\mu)$ as a function of inertia number $(I)$ is another rheological model that has been widely investigated for granular flow modeling. In this model, the inertia number is defined as:

$$
\dot{\gamma} d_{p} / \sqrt{\sigma / \rho_{p}},
$$

in which $d_{p}$ and $\rho_{p}$ are the diameter and density of particles, respectively, and $\sigma$ is the normal stress. The applicability of this rheology has been evaluated through different experiments and simulations for different materials and configurations [7,8]. As a classical benchmark for the study of granular flows, the $\mu(I)$ rheology was used to simulate the collapse of the dry granular columns, the results of which were in good agreement with the experimental observations, both in $2 \mathrm{D}[9,10]$ and $3 \mathrm{D}[10,11]$ numerical simulations. However, to describe the flow of wet granular materials, a rheological model with the consideration of the effect of liquid saturation should be developed.

To study the rheological behavior of wet granular materials, a numerical method capable of modeling the interaction between water and particles must be employed. Through a Discrete Element Method (DEM) [12] used in this study, grains are described as discrete particles and the particle motion is modeled through Newton's second law with a contact model. This method can also be utilized to establish a rheological equation through the numerical modeling of the shear flows. The stresses and velocity fields obtained from the rheology of shear flows were validated by the experimental observations $[13,14]$. The effects of interparticle contact properties (friction, cohesion, and softness) [15] and particle shape $[16,17]$ on the rheological behavior were also investigated in the literature. Since DEM functions based on the action of interparticle forces, water-induced inter-particle forces must be determined first. In this regard, to model the motion of particles in wet condition, the buoyancy and surface tension forces must be calculated for each saturation.

How water positions itself between particles controls the inter-particle forces. Water distribution which varies with saturation is an important factor in determining the rheological behavior of a wet granular material [18]. The commonly used pendular bridge model [19] can illustrate the liquid distribution and inter-particle forces in partially wetted conditions regardless of the exact amount of saturation. Waterinduced inter-particle forces and their dependence on the bridge volume and rupture distance (i.e., a critical inter-particle distance for which a liquid bridge exists) were investigated for equal-sized [20,21] and unequalsized $[22,23]$ spherical particles in the pendular state.
This model was also used in the DEM simulations which was validated by experimental results [24,25]. However, to obtain the rheological behavior over a wide range of saturations, it is essential that the water distribution remain correlated with saturation and, then, the inter-particle forces be calculated.

The main objective of this study was to obtain the rheology of a wet granular material for different saturations. To this end, first, a model is developed that can formulate the distribution of water between particles (Subsection 2.2) and then, the resulting forces are calculated for different saturations (Subsection 2.3). The numerical method is presented in Section 3. A DEM open-source code, LIGGGTHS, is used to construct a $3 \mathrm{D}$ shear cell as a numerical rheometer to investigate the rheological behavior of the dry and wet granular materials (Subsection 4.1). To evaluate the performance of the proposed model, the collapse of two-dimensional granular material on a horizontal rigid bed is simulated using the obtained rheological model (Subsection 4.2).

\section{Physical background}

Considering soil as a granular material, this section begins by describing the assumptions regarding granular soil, theoretical modeling of water distribution, and its associated inter-particle forces.

\subsection{Description of granular material}

The granular soil is considered to be a homogenous assembly of mono-sized spherical particles. This paper considers a cubic lattice initial packing for the granular material (Figure 1). This simplification makes it possible to construct a theoretical model for determining the distribution of water between particles in different saturations. The impact of other arrangements (for example, faced-centered cubic lattice) on the results could be a research topic in the future.

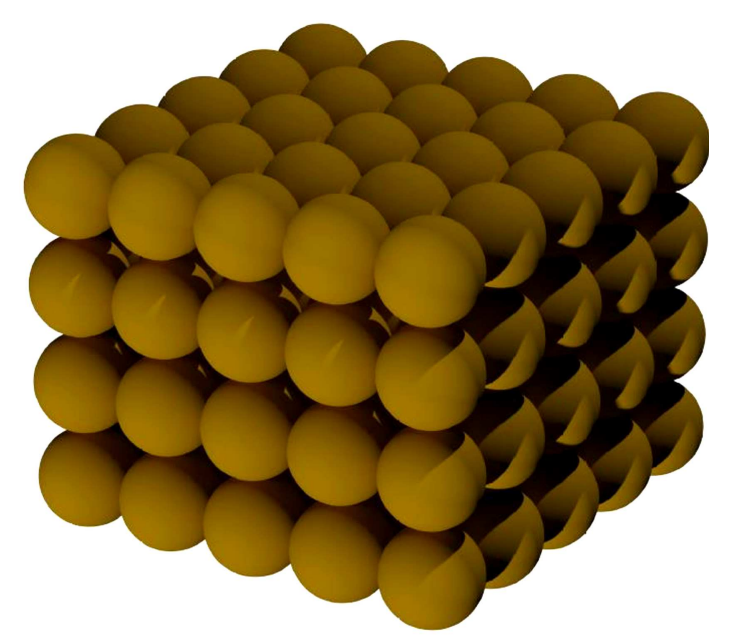

Figure 1. Initial arrangement of particles. 


\subsection{The distribution of water between particles}

In this study, similar to the rainfall infiltration, water content (saturation) is considered to decrease with depth. In a typical infiltration study, water saturation is computed at different depths and times. This is the starting point of the current study where one is interested in the instantaneous rheology of the local wet material.

Figure 2 describes a column of soil particles (as a water passage) and different zones (A to I) which can be distinguished within the material. All pores are filled with water in the near-surface zone (A saturated zone) and during infiltration, some air is trapped in the spaces between particles (zones B to D) [26]. In fact, below the saturated zone, there are some semi-saturated zones whose difference is the amount of trapped air (zones B to D - semi-saturated zones). In zones A to $\mathrm{D}$, pore water pressure $(u)$ is hydrostatic and positive. In these saturated and semi-saturated zones, the buoyancy effect of water reduces the normal stress to effective stress $\left(\sigma^{\prime}\right)$ according to Terzaghi's principle as follows:

$$
\sigma^{\prime}=\sigma-u
$$

which has a large impact on the behavior of the material. For example, in such zones in the slopes, water tends to destabilize the material because the reduction in normal stress lowers frictional resistance to sliding that is $\sigma^{\prime} \tan \varsigma$ ( $\varsigma$ is the angle of internal friction), which can cause slope instability [5].

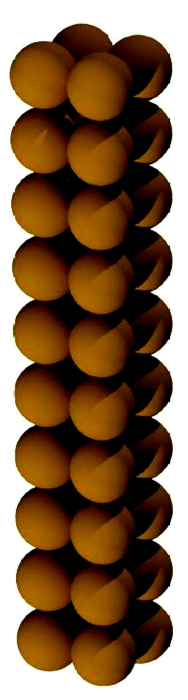

(a)

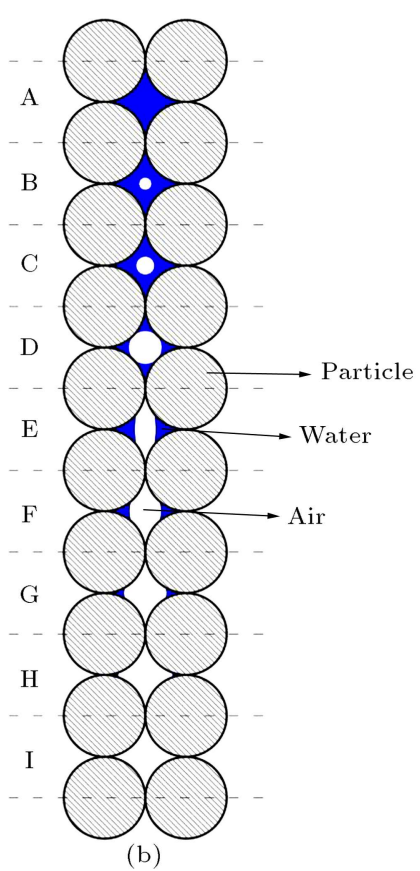

(b)
Figure 2. (a) A column of soil particles. (b) Different zones during rainfall infiltration: saturated (A), semi saturated (B to D), unsaturated (E to H), and dry (I).
Under semi-saturated zones, due to the interfacial tension effect, water is drawn into the smaller spaces between particles (zones $\mathrm{E}$ to $\mathrm{H}$ - unsaturated zones) and as the depth increases, the volume of trapped water decreases. In these zones, the interaction between particles, water and air develops a state that yields negative pore water pressure. This negative pore pressure causes greater effective stress than the normal stress (according to Bishop's equation [5]), which increases the frictional resistance force to sliding. So, in the unsaturated zone, the overall shear strength of material increases compared to the dry state.

In fact, during rainfall infiltration, all three zones (saturated or semi-saturated, unsaturated and dry) exist. In each zone, the water-induced forces are different and to calculate these forces, the distribution of water between particles must be determined. To formulate this distribution, some geometric parameters must be introduced first (Figure 3 ).

Contact Angle $(\theta)$ is the angle between the solid surface and the tangent of the water-air interface, conventionally measured through the water. In this work, the contact angle is assumed constant.

Meniscus Curvature Radius $(r)$ is the radius of curvature at the interface between water and air. In the present study, the toroidal approximation [19] is applied which assumes that the meridian profile of the liquid-air interface has a circular shape. So, the meniscus radius of curvature is obtained as follows:

$$
r(R, \phi, \theta)=2 R \cdot\left[\frac{\sin ^{2}(\phi / 2)}{\cos (\phi+\theta)}\right] .
$$

Figure 4 shows the schematic of inter-particle water. Approximating the bridge as a cylinder and subtracting two spherical caps from its top and bottom, the bridge volume is obtained as follows:

$$
\begin{aligned}
V_{W} \simeq & \pi R^{2} \sin ^{2} \phi \times 2 R(1-\cos \phi)-2 \\
& \times \frac{\pi}{3} R^{3}(2+\cos \phi)(1-\cos \phi)^{2} .
\end{aligned}
$$

The space between four particles and the percentage of its occupation by water was considered as the criterion for calculating the saturation (according to Figure 5). The saturation degree $(S)$ in each zone of Figure 2 can be found by dividing the volume of water by the inter-particle volume. From zone A to $\mathrm{D}$, trapped air, as an air core, is created in the space between particles and as its diameter increases, saturation decreases. In zone D $(S=34.2 \%)$, the air core reaches the surface of particles.

According to Figure 5, for zones $\mathrm{E}$ to $\mathrm{H}$, there are twelve quarters of bridge volumes between particles and the saturation is calculated by Eq. (4) is shown in Box I.

As it can be seen, that saturation is independent of the particle size. 


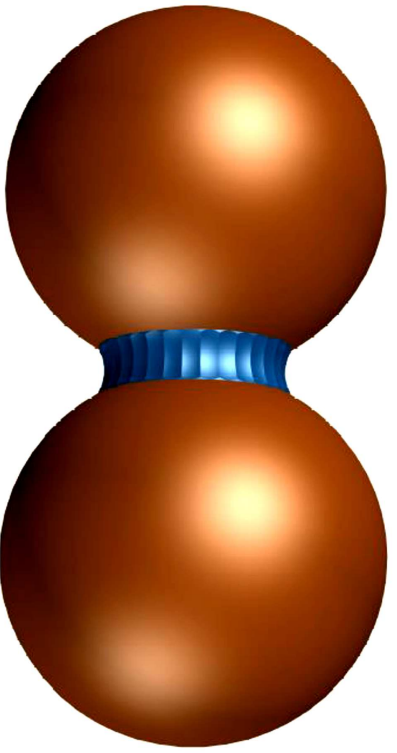

(a)

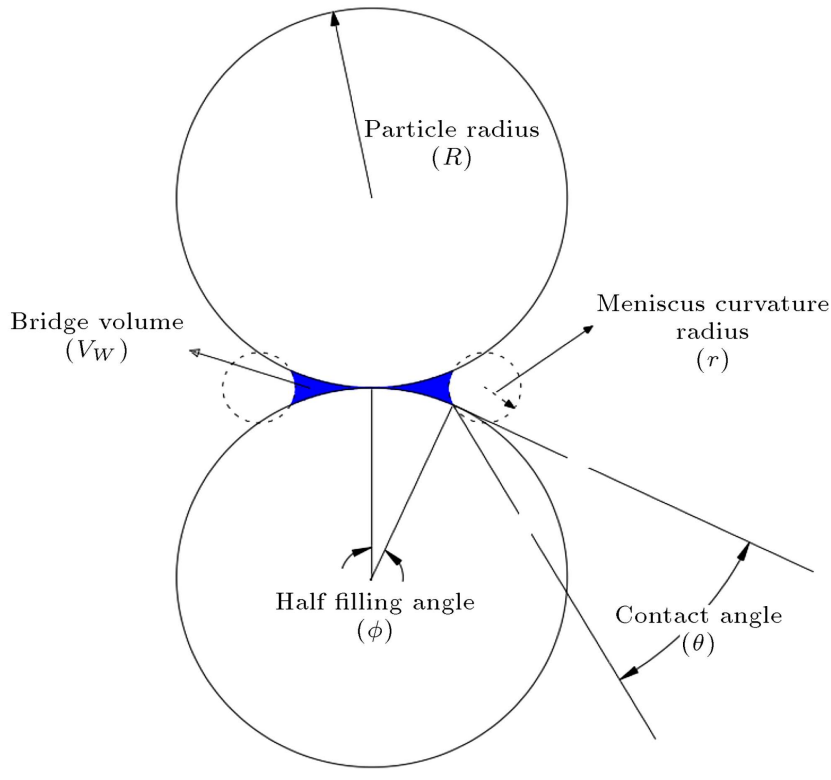

(b)

Figure 3. (a) Schematic of a water bridge between two spherical particles and (b) parameters required to formulate the distribution of water between particles.

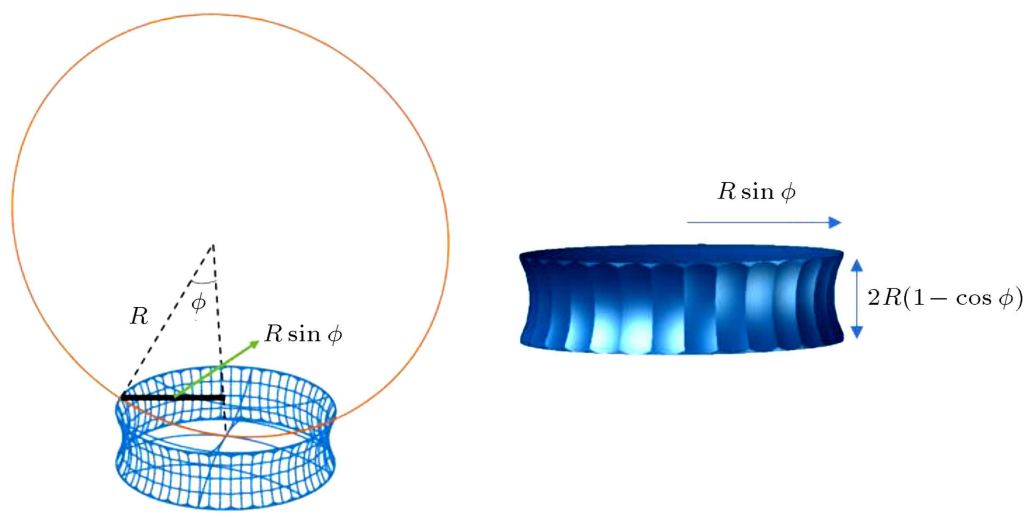

Figure 4. Schematic of the water bridge and its dimensions.

$$
\begin{aligned}
S & =\frac{12 \times\left(\frac{V_{W}}{4}\right)}{V_{\text {Pore }}}=3 \times \frac{\pi R^{2} \sin ^{2} \phi \times 2 R(1-\cos \phi)-2 \times \frac{\pi}{3} R^{3}(2+\cos \phi)(1-\cos \phi)^{2}}{8 R^{3}-8 \times \frac{1}{8} \times \frac{4}{3} \pi R^{3}} \\
& =3 \times \frac{\pi \sin ^{2} \phi \times 2(1-\cos \phi)-2 \times \frac{\pi}{3}(2+\cos \phi)(1-\cos \phi)^{2}}{8-\frac{4}{3} \pi} .
\end{aligned}
$$

Box I

For $S<34.2 \%$, saturation decreases by depth as half filling angle reduces. Figure 6 shows variation in saturation due to the half filling angle.

\subsection{Calculation of the water induced forces}

The water-induced forces are the buoyancy $\left(F_{B}\right.$, for zones A to D) and capillary forces $\left(F_{C a p}\right.$, for zones E to $\mathrm{H})$. The capillary force can be considered as having two components [19]: a pressure term (due to YoungLaplace pressure) and another component due to the direct impact of surface tension. The pressure term is calculated through the Young-Laplace equation, which 


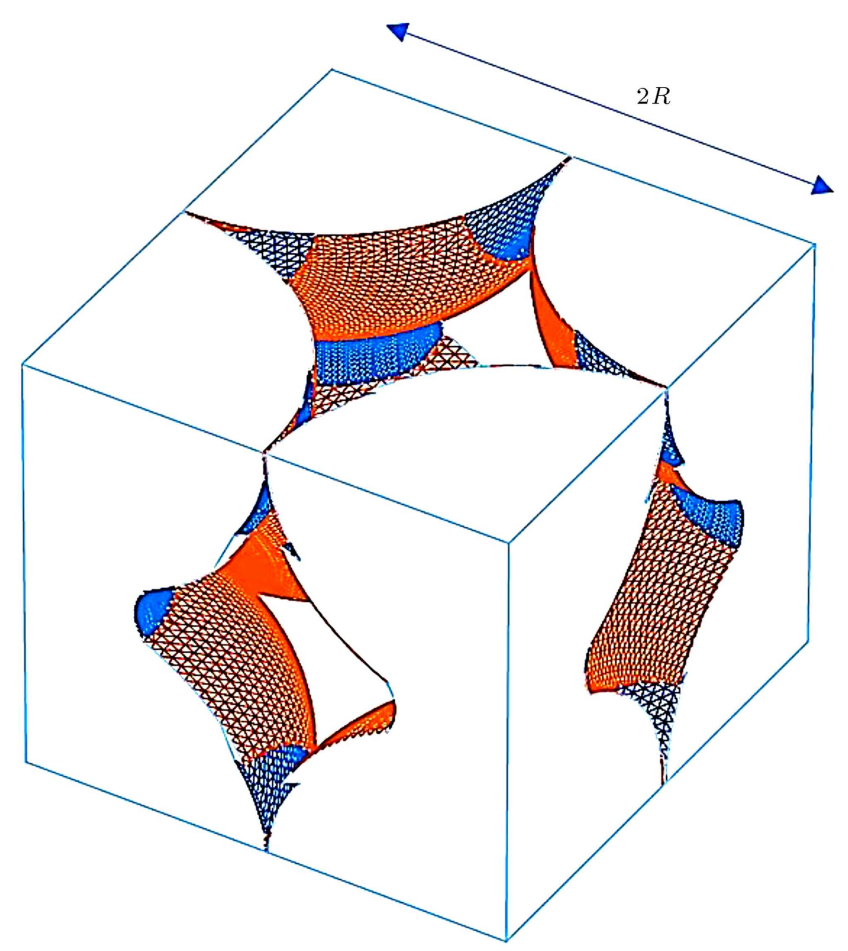

Figure 5. The space between four particles where the saturation is calculated.

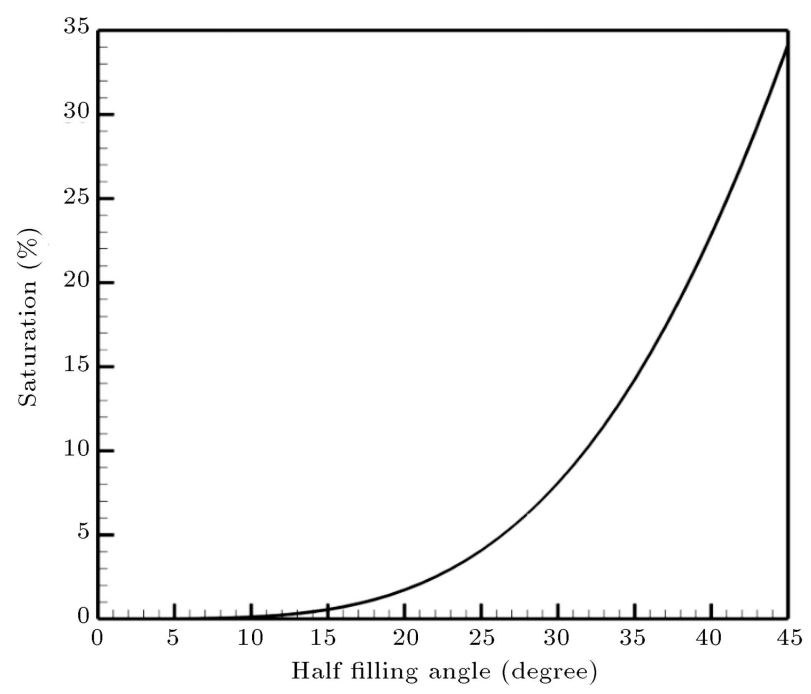

Figure 6. Variation of saturation with the half filling angle for $\theta=30^{\circ}$ (zones E to I).

relates the mean curvature of the water surface to the pressure difference between the water and the outside air $(\Delta P)$. In the three-dimensional form, this relationship is written as follows:

$$
\Delta P=\frac{2 \psi_{W A}}{r},
$$

where $\psi_{W A}$ is water-air interfacial tension. The pressure term is obtained from the product of the pressure difference in the projection of the wetted area in the

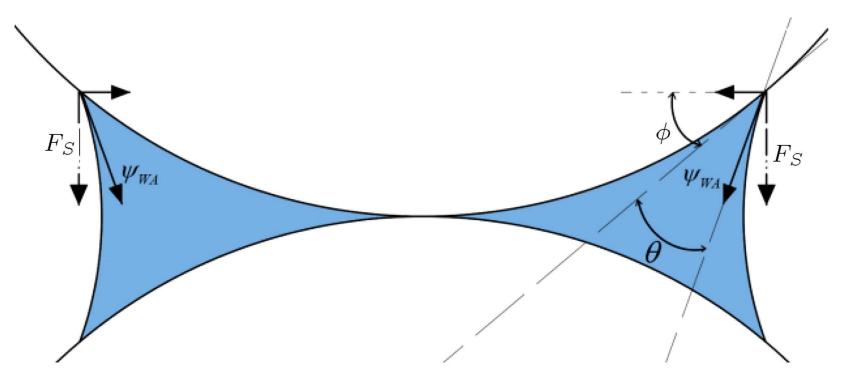

Figure 7. Components of surface tension force.

particle $\left(A_{W e t}\right)$. According to Figure $4, A_{W e t}$ is a circle with radius $R \sin \phi$. Therefore, the pressure term of capillary force is:

$$
F_{\Delta P}=\Delta P \cdot A_{w e t}=\frac{2 \psi_{W A}}{r} \cdot \pi R^{2} \sin ^{2} \phi .
$$

The surface tension term, applied to each particle, has two components in horizontal and vertical directions (Figure 7). The vertical component creates an attraction between particles and the magnitude of this attractive force is:

$$
\begin{aligned}
F_{S} & =\psi_{W A} \cos \left(\frac{\pi}{2}-\phi-\theta\right) \cdot 2 \pi R \sin \phi \\
& =2 \pi R \psi_{W A} \sin \phi \cos \left(\frac{\pi}{2}-\phi-\theta\right),
\end{aligned}
$$

which lies along the line joining the centers of two particles. So, the magnitude of capillary force is:

$$
\begin{aligned}
F_{C a p}= & F_{\Delta P}+F_{S}=\frac{2 \psi_{W A}}{r} \cdot \pi R^{2} \sin ^{2} \phi \\
& +2 \pi R \psi_{W A} \sin \phi \cos \left(\frac{\pi}{2}-\phi-\theta\right) .
\end{aligned}
$$

The surface tension force between two particles exists until the distance between the center of particles reaches a critical distance $\left(\delta_{\text {crit }}\right)$ [27] and the liquid bridge ruptures. In this study, the critical distance is considered to be [19]:

$$
\delta_{c r i t}=V_{W}^{1 / 3} \cdot\left(1+\frac{\theta}{2}\right) .
$$

In the current study, the changes in the bridge shape and capillary forces due to the changes in the inter-particle distance are not considered until reaching the critical distance.

The buoyancy force for each particle is calculated as:

$$
F_{B}=A_{\text {particle }} \cdot \rho_{\text {water }} \cdot g .
$$

These fluid induced forces are later used to determine shear forces between the DEM particles.

It should be noted that the particles are not 
always in the same position shown in Figure 1. Particles can move if a shear force is applied to them. During the particle movements, in saturated and semisaturated states (A to D), the water-induced force, i.e., buoyancy force, remains unchanged. For states $\mathrm{E}$ to $\mathrm{H}$, as an assumption, changes in the capillary forces during particle motion have been ignored until the critical inter-particle distance is reached, which destroys these forces.

\section{Numerical modeling}

To construct a numerical rheometer, the DEM is used. Since its introduction by Cundall and Strack [12], the DEM has been used broadly to simulate granular materials.

\subsection{Governing equations}

In DEM, particles are rigid bodies with very small inter-particle deformations that can be simplified as inter-particle overlaps [28]. In this method, the motion of each particle is tracked in space and time throughout the computational domain by solving Newton's second law of motion (according to Figure 8):

$$
\begin{aligned}
& m_{i} \frac{d^{2} \vec{x}_{i}}{d t^{2}}=\sum_{\{i, j\}=c} \vec{F}_{i j}+m_{i} \vec{g}+\vec{F}_{B}, \\
& I_{i} \frac{d \vec{\omega}_{i}}{d t}=\sum_{\{i, j\}=c} \vec{d}_{c j i} \times \vec{F}_{i j},
\end{aligned}
$$

where $m_{i}, \vec{x}_{i}, I_{i}$, and $\vec{\omega}_{i}$ are mass, position vector, the moment of inertia, and angular velocity of the particle

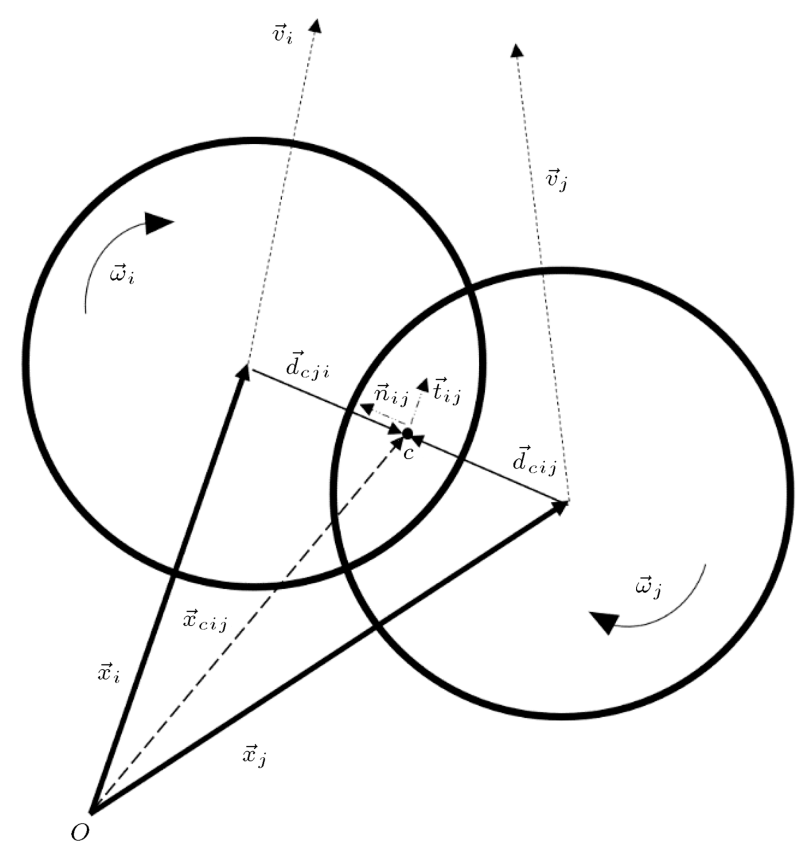

Figure 8. Parameters and variables used for interaction of particles $i$ and $j$. $i$, respectively. The right-hand side of Eq. (11) is the sum of forces acting on particle $i$ resulting from interparticle forces $\left(\vec{F}_{i j}\right)$, the gravitational force $\left(m_{i} \vec{g}\right)$, and the buoyancy force $\left(\vec{F}_{B}\right)$. The inter-particle forces consist of two terms: collision-induced force $\left(\vec{F}_{c i j}\right)$ and capillary force $\left(\vec{F}_{C a p}\right)$. The right-hand side of Eq. (12) also indicates the torque on particle $i$ induced by the collision with particle $j . \quad \vec{d}_{c i j}$ indicates the distance vector from the center of the particle to the contact point of two particles $(c)$. The contact point with the position vector of $\vec{x}_{c i j}$ is defined to be at the center of the overlapped volume. The collision-induced force $\left(\vec{F}_{c i j}\right)$ is defined using force-overlap relationships and is written at the contact point as [28]:

$$
\vec{F}_{c i j}=F_{c i j}^{n} \vec{n}_{i j}+F_{c i j}^{t} \vec{t}_{i j}
$$

where $\vec{n}_{i j}, \vec{t}_{i j}, F_{c i j}^{n}$, and $F_{c i j}^{t}$ are normal and tangent unit vectors as well as normal and tangent forces at the contact point $c$, respectively.

For particles $i$ and $j$ with radius $R$ that move with linear $\left(\vec{v}_{i}, \vec{v}_{j}\right)$ and angular $\left(\vec{\omega}_{i}, \vec{\omega}_{j}\right)$ velocities, a collision will be detected if they have a positive overlap value $(\delta)$ by the following inequality:

$$
\delta=2 R-\left(\vec{x}_{i}-\vec{x}_{j}\right) \cdot \vec{n}_{i j}>0 .
$$

Then, the normal contact force consisting of a linear repulsion term and a linear dissipation term is considered as follows [28]:

$$
F_{c i j}^{n}=k_{n} \delta+\beta_{n}\left(\vec{V}_{i j} \cdot \vec{n}_{i j}\right)
$$

where $k_{n}$ and $\beta_{n}$ are normal stiffness and damper coefficients. $\quad \vec{V}_{i j}$ is the velocity vector of a particle $i$ relative to particle $j$ :

$$
\vec{V}_{i j}=\left(\vec{v}_{i}+\vec{\omega}_{i} \times \vec{d}_{c j i}\right)-\left(\vec{v}_{j}+\vec{\omega}_{j} \times \vec{d}_{c i j}\right)
$$

where:

$$
\vec{d}_{c j i}=-\vec{d}_{c i j}=\vec{x}_{c i j}-\vec{x}_{i}=-\left(R-\frac{\delta}{2}\right) \vec{n}_{i j}
$$

The tangential contact force is also calculated by the following equation [28]:

$$
F_{c i j}^{t}=k_{t} \lambda+\beta_{t}\left(\vec{V}_{i j} \cdot \vec{t}_{i j}\right)
$$

where $k_{t}$ and $\beta_{t}$ are tangential stiffness and damper coefficients. $\lambda$ is the tangential displacement of the contact point and is defined as [28]:

$$
\lambda=\int_{t=t_{0}}^{t=t}\left(\vec{V}_{i j} \cdot \vec{t}_{i j}\right) d t
$$


where $t_{0}$ is the time when the two particles first come into contact and $t$ is the current simulation time.

To determine the coefficients $\left(k_{n}, \beta_{n}, k_{t}, \beta_{t}\right)$, the non-linear Hertz contact model [28] is used. In this model:

$$
\begin{array}{rlrl}
k_{n} & =\frac{4}{3} E^{*} \sqrt{R^{*} \delta}, & \frac{1}{E^{*}} & =\frac{\left(1-v_{i}^{2}\right)}{E_{i}}+\frac{\left(1-v_{j}^{2}\right)}{E_{j}}, \\
\frac{1}{R^{*}}=\frac{1}{R_{i}}+\frac{1}{R_{j}}, & \beta_{n}=2 \sqrt{\frac{5}{6} \alpha \sqrt{S_{n} m^{*}}} \\
\frac{1}{m^{*}}=\frac{1}{m_{i}}+\frac{1}{m_{j}}, & \alpha=\frac{\ln \varepsilon}{\sqrt{\ln ^{2} \varepsilon+\pi^{2}}}, \\
S_{n}=2 E^{*} \sqrt{R^{*} \delta_{n}}, & \frac{1}{G^{*}}=\frac{\left(2-v_{i}\right)}{G_{i}}+\frac{\left(2-v_{j}\right)}{G_{j}} \\
k_{t}=8 G^{*} \sqrt{R^{*} \delta_{n}}, & \beta_{t}=2 \sqrt{\frac{5}{6}} \alpha \sqrt{k_{t} m^{*}}
\end{array}
$$

where $E, G, v$, and $\varepsilon$ are Young's modulus, shearing modulus, Poisson's ratio, and restitution coefficient of particles. Note that the tangential force is calculated with Eq. (18) when it is less than the Coulomb friction force. In other words:

$$
\begin{aligned}
& F^{t}{ }_{c i j}= \\
& \left\{\begin{array}{l}
k_{t} \lambda+\beta_{t}\left(\vec{V}_{i j} \cdot \vec{t}_{i j}\right) \text { if } \quad k_{t} \lambda+\beta_{t}\left(\vec{V}_{i j} \cdot \vec{t}_{i j}\right) \leq \mu_{C} F^{n}{ }_{c i j} \\
\mu_{C} F^{n}{ }_{c i j} \quad \text { if } \quad k_{t} \lambda+\beta_{t}\left(\vec{V}_{i j} \cdot \vec{t}_{i j}\right)>\mu_{C} F^{n}{ }_{c i j}(20)
\end{array}\right.
\end{aligned}
$$

where $\mu_{C}$ is the Coulomb friction coefficient. In this study, $\mu_{C}$ is assumed to be equal to $\tan \varsigma$ and $\varsigma$ is the internal friction angle of the granular material, as mentioned before.

The inter-particle water-induced forces are also computed as:

$$
\begin{aligned}
& \vec{F}_{C a p}=\left\{\begin{array}{ll}
-F_{C a p} \vec{n}_{i j} & \text { if } \quad 0<S<34.2 \% \\
0 & \text { if } \quad S \geq 34.2 \%
\end{array},\right.
\end{aligned}
$$

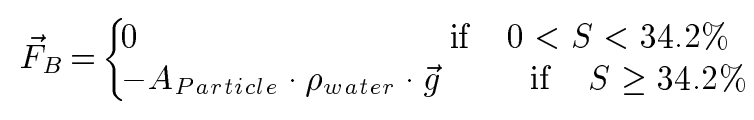

In DEM, the numerical simulation starts from an assembly of particles with the specified size, material properties, initial positions, and velocities. The interparticle forces and moments are obtained from initial conditions and then, substituted into Eqs. (11) and (12) to obtain the updated positions and velocities by marching in time. Spatial positions, velocities (linear and angular), and applied forces and torques for each particle as functions of time are the typical results of a DEM simulation. These data can be used to investigate the macroscopic behavior of the granular material.

The equations mentioned above were implemented in LIGGGHTS, which is an open-source DEM simulation code used for the simulation of granular materials [29]. The non-linear Hertz contact model is included in LIGGGHTS and can be utilized to simulate dry granular materials. By applying this model, all equations mentioned above, excluding Eqs. (21) and (22), are employed.

For the simulation in the wet condition, the nonlinear Hertz contact model is modified by adding the capillary force (Eq. (21)) as a constant value to the inter-particle forces, for $S<S_{\text {crit }}$. For this range of saturations, when two particles are in physical contact $(\delta>0)$, the particle interaction will be equal to the sum of the collision-induced $\left(\vec{F}_{c i j}\right)$ and the capillary forces $\left(\vec{F}_{C a p}\right)$. When two particles are not in physical contact but within the rupture distance $(\delta<0$ and $\left.|\delta| \leq \delta_{\text {crit }}\right)$, the capillary force is the only inter-particle force. When the inter-particle distance is greater than the rupture distance $\left(\delta<0\right.$ and $\left.|\delta|>\delta_{\text {crit }}\right)$, there is no inter-particle force. It can be written as:

$$
\vec{F}_{i j}= \begin{cases}\vec{F}_{c i j}+\vec{F}_{C a p} & \text { if } \delta>0 \\ \vec{F}_{C a p} & \text { if } \delta<0 \text { and }|\delta| \leq \delta_{\text {crit }} \\ 0 & \text { if } \delta<0 \text { and }|\delta|>\delta_{\text {crit }}\end{cases}
$$

The buoyancy force (Eq. (22)) is also added as a body force acting on the computational domain for $S \geq S_{\text {crit }}$.

Constructing a numerical rheometer and utilizing that for obtaining the saturation-based rheological model are described in the results.

\section{Results}

The results are divided into two parts: obtaining the saturation-based rheological model and collapse simulation of the granular material at different saturations using the saturation-based rheological model.

\subsection{The rheological model}

In this section, a three-dimensional numerical rheometer is constructed and the rheological model is obtained for the granular material at different saturation levels.

\subsubsection{Setup of a shear cell}

To find the rheological behavior of a specimen, it is sheared under different normal and shear stresses and its apparent viscosity can be obtained as a function of shear rate and normal stress at different saturations. For this purpose, a 3D shear cell as a numerical rheometer is constructed using the DEM specimen which is a monodisperse assembly of spherical particles (as schematically shown in Figure 9(a)). All particles are positioned in a cubic lattice form inside a cube made of two frictional rigid walls and four virtual walls as periodic boundaries (as shown in Figure 9(b)).

The bottom wall is fixed and the top wall is under a downward force exerting constant normal 


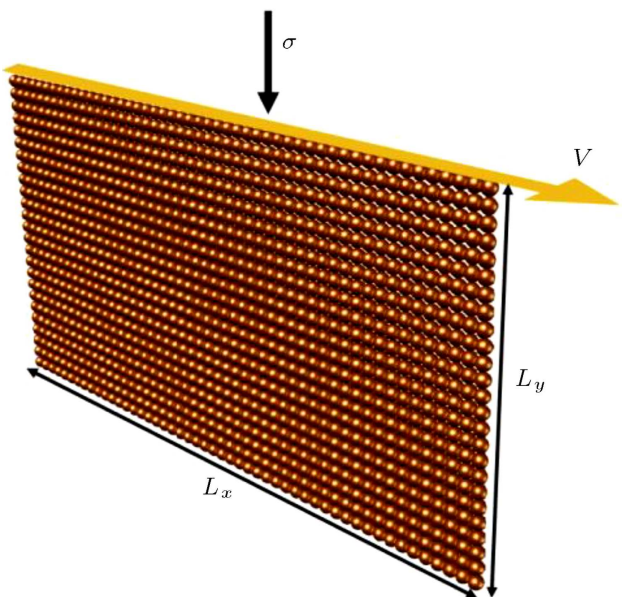

(a)

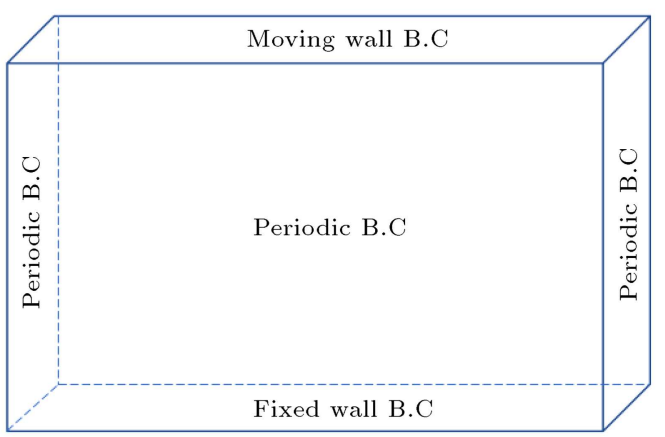

(b)

Figure 9. (a) Schematic of a shear cell and (b) its boundary conditions.

stress. Shear stress is applied to the specimen while the top wall moves at a constant speed. Periodic boundary conditions are applied to the lateral sides of the computational domain. To impose wall shear stress, top and bottom walls are made of the same particles as the flowing. This creates a reasonably rough surface representing real conditions. In the following, this configuration can be used to study both dry and wet conditions.

\subsubsection{Dry rheological model}

A specimen of dry sand with characteristics shown in Table 1 is studied first. The shear cell contains 1250 particles and the other parameters are $\sigma=1 \mathrm{kPa}, V=$ $21.46 \mathrm{~mm} / \mathrm{s}, L_{x}=50 \mathrm{~mm}$, and $L_{y}=25 \mathrm{~mm}$. The shearing process begins with velocity $V$ for the top wall and a linear velocity profile is assumed as an initial condition [30].

To describe the material rheological behavior, the apparent viscosity, $\mu=\tau / \dot{\gamma}$, is used. The shear stress is calculated by dividing the resulting force applied on the top wall by its length $\left(L_{x}\right)$. Also, the shear rate is equal to the ratio of top wall velocity to the width of the shear layer as $\dot{\gamma}=V / L_{y}$.

An important point to be considered is the de-

Table 1. Specimen specifications.

\begin{tabular}{cc}
\hline Specification & Value \\
\hline Particle radius $(R)$ & $0.5 \mathrm{~mm}$ \\
Particle density $(\rho)$ & $2660 \mathrm{~kg} / \mathrm{m}^{3}$ \\
Porosity $(\kappa)$ & 0.476 \\
Young's modulus $(E)$ & $50 \mathrm{MPa}$ \\
Poisson ratio $(v)$ & 0.2 \\
Angle of internal friction $(\zeta)$ & $34^{\circ}$ \\
Coefficient of restitution $(\varepsilon)$ & 0.56 \\
Particle - water contact angle $(\theta)$ & $30^{\circ}$ \\
\hline
\end{tabular}

pendence of the results on the specimen size. For this purpose, three specimens with different particle numbers are studied in shear cells with different dimensions, according to Table 2. The computed apparent viscosities are shown in Figure 10. As it can be seen, the results become independent of the specimen size by increasing the number of particles to more than those in Case \#2. Figure 10 also indicates that the viscosity of dry sand decreases as the shear rate increases.

Table 2. Three specimens with different particle numbers.

\begin{tabular}{cccc}
\hline Case & $\begin{array}{c}\text { Number of } \\
\text { particles }\end{array}$ & $\boldsymbol{L}_{\boldsymbol{x}}(\mathbf{m m})$ & $\boldsymbol{L}_{\boldsymbol{y}}(\mathbf{m m})$ \\
\hline 1 & 300 & 25 & 12 \\
2 & 1250 & 50 & 25 \\
3 & 5000 & 100 & 50 \\
\hline
\end{tabular}

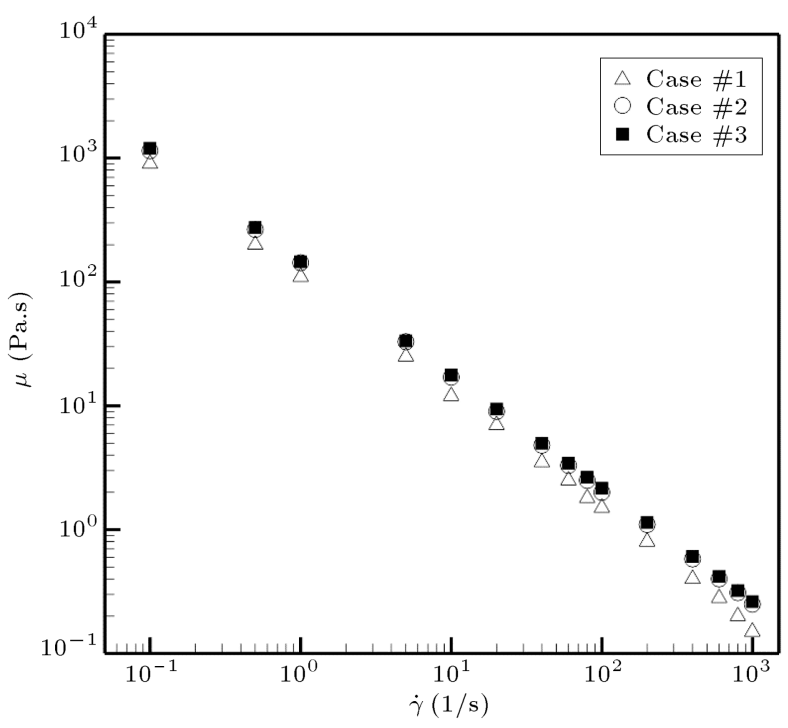

Figure 10. Apparent viscosity data for three specimens with different particle numbers and $\sigma=1 \mathrm{kPa}$. 


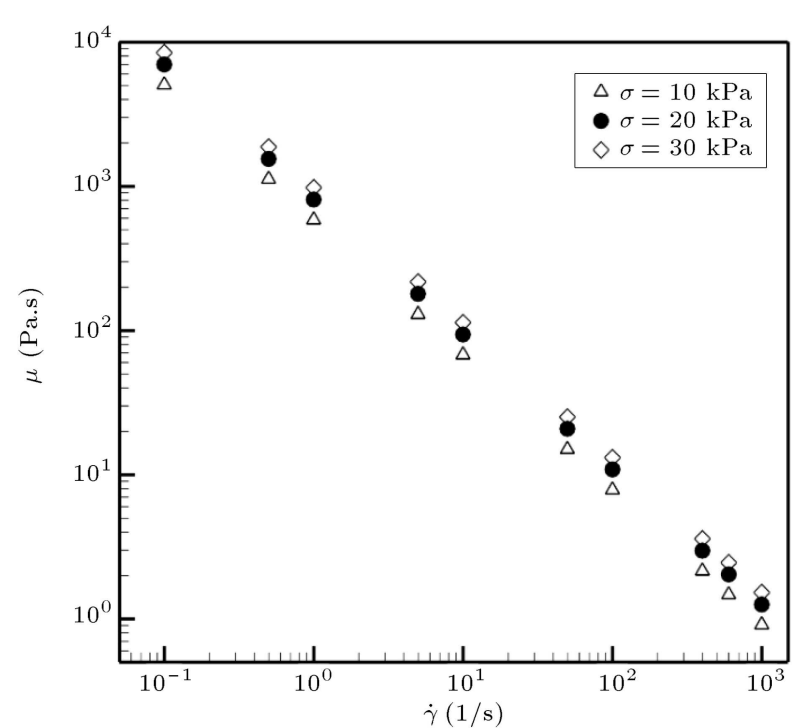

Figure 11. Variation of apparent viscosity with shear rate for three different applied normal stresses - the dry specimen.

This behavior (i.e., reducing viscosity with increasing shear rate) is consistent with the experimental results obtained by Schwarze et al. [24] for a different sand specimen.

Figure 11 shows the variation of apparent viscosity with the shear rate in three different normal stresses for the dry specimen. It is observed that increasing the normal stress increases the viscosity at any shear rate. The rheological equation which relates apparent viscosity, shear rate, and normal stress can be extracted as follows for the dry specimen (where $\sigma$ is measured in $\mathrm{Pa})$ :

$$
\mu[\text { Pa.s }]=0.49\left(\frac{\dot{\gamma} d}{\sqrt{\sigma / \rho}}\right)^{-0.936}=0.49 I^{-0.936}
$$

where $d$ and $\rho$ are the diameter and density of specimen particles, and the inertia number is defined as $I=$ $\frac{\dot{\gamma} d}{\sqrt{\sigma / \rho}}[31]$.

\subsubsection{Wet rheological model}

Figure 12 shows apparent viscosity variation with a saturation degree at $I=8 \times 10^{-3}$. This figure indicates that the viscosity of the wet specimen increases with saturation, up to a critical saturation degree $\left(S_{\text {crit }}=\right.$ $34.2 \%$ ) and its value is higher than that of the dry one. In fact, below this critical saturation, the effect of water-induced forces adds cohesion to the specimen. When the saturation degree exceeds $S_{\text {crit }}$, the viscosity of the wet specimen becomes lower than that of the dry specimen. This behavior can be attributed to the elimination of the surface tension force between particles and the repulsive effect of the buoyancy force that makes the specimen less resistant to shearing.

Iverson et al. [32] investigated experimentally the

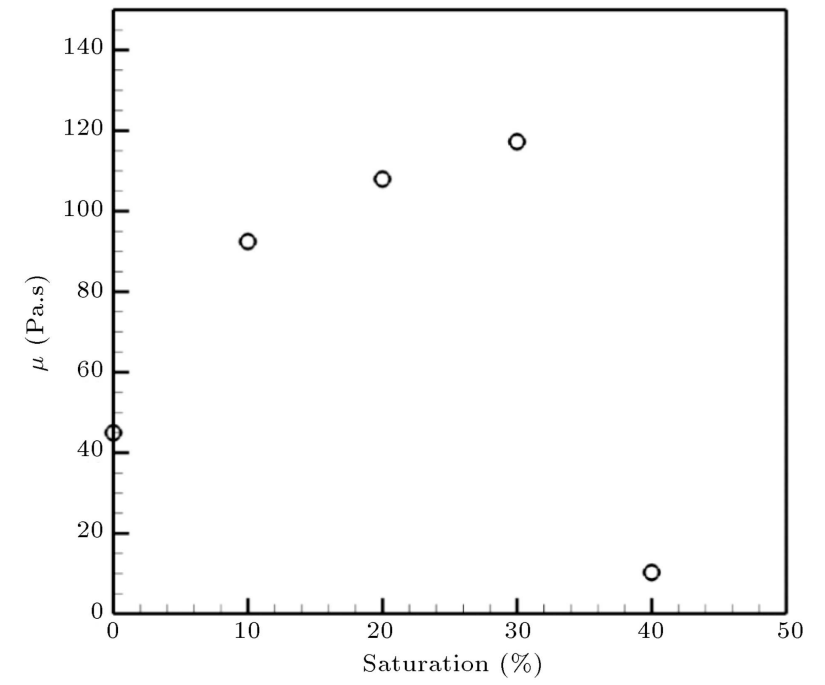

Figure 12. Variation of apparent viscosity with saturation for $I=8 \times 10^{-3}$.

effect of soil bed water content on the growth of momentum of the debris flow. In their experiments, the bed porosity was about 0.4 and the debris flow was saturated. They changed the saturation of the bed and measured the momentum of the flowing material. Their experiments showed that up to $S_{\text {bed }} \simeq 55 \%$, momentum of the flowing material decreased with increasing bed saturation. However, when the bed saturation exceeds $S_{\text {bed }} \simeq 55 \%$, the momentum of debris flow increases with increasing bed saturation. In fact, in their experiments, the sign of pore water pressure changes from negative to positive at $S_{\text {bed-crit }} \simeq 55 \%$. One of the reasons for the higher critical saturation degree in their experiments compared to the material investigated in this work can be the difference in the material porosity.

To obtain a rheological model for the specimen, simulations were performed for different applied normal stresses and shear rates. Figure 13 shows the variation of apparent viscosity with inertia numbers at various saturation levels. As can be seen, as the inertia number increases, the difference between the apparent viscosities for different saturations decreases.

From Figure 13, the general rheological equation for the wet specimen can be formulated as follows:

$$
\mu[\mathrm{Pa} . \mathrm{s}]=A \times I^{-B},
$$

where $A$ and $B$ values are listed in Table 3 . By applying Eq. (25) along with Table 3, the flow of wet granular materials can be studied.

\subsection{Granular collapse simulation using the rheological model}

In this section, the rheological relations obtained above were implemented into the SePeHr [33] code, an inhouse flow solver, which is based on numerical simulation of granular flows in a continuum context using Incompressible Smoothed Particle Hydrodynamics 


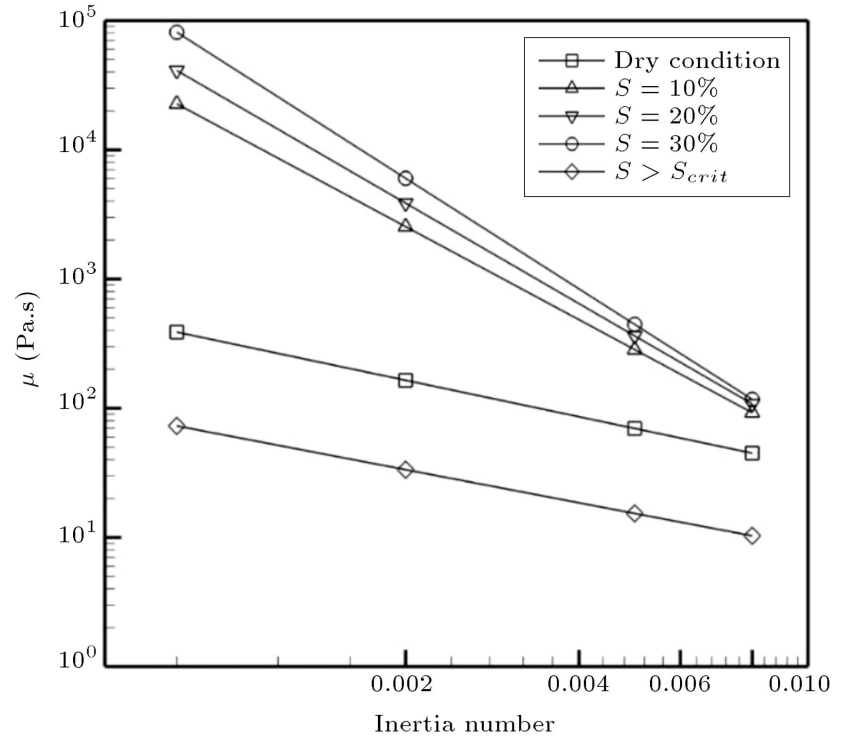

Figure 13. Variation of apparent viscosity with inertia number for different saturation levels.

Table 3. Coefficients of the rheological equation for different saturations.

\begin{tabular}{|c|c|c|c|c|c|}
\hline \multirow[b]{2}{*}{ Coefficient } & \multicolumn{5}{|c|}{ Saturation degree } \\
\hline & $10 \%$ & $20 \%$ & $30 \%$ & $S$ & $\geq S_{c r i t}$ \\
\hline$A$ & $9 \mathrm{e}-4$ & $4.2 \mathrm{e}-4$ & $1.3 \mathrm{e}-4$ & & 0.17 \\
\hline$B$ & 2.39 & 2.58 & 2.84 & & 0.85 \\
\hline
\end{tabular}

Table 4. Specimen specifications in Nguyen et al. experiments [35].

\begin{tabular}{cc}
\hline Specification & Value \\
\hline Density $(\rho)$ & $20.4 \mathrm{kN} / \mathrm{m}^{3}$ \\
Young's modulus $(E)$ & $5.84 \mathrm{MPa}$ \\
Poisson ratio $(v)$ & 0.3 \\
Angle of internal friction $(\zeta)$ & $21.9^{\circ}$ \\
\hline
\end{tabular}

method (ISPH) [34]. This code has been recently used for modeling the collapse of two-dimensional dry granular materials using visco-plastic rheology on horizontal rigid and entrainable beds [33]. In the following, first, the validity of the rheological model in the dry state is examined by comparing the simulation with experimental results and, then, the simulation is performed in the case of different saturations.

\subsubsection{Dry granular material}

To conduct this verification, the collapse of a twodimensional granular material on a horizontal rigid bed, as a well-defined benchmark problem, is compared with the experimental results obtained by Nguyen et al. [35]. Figure 14 schematically shows the case studied in this section and the properties of the specimen are listed in Table 4.

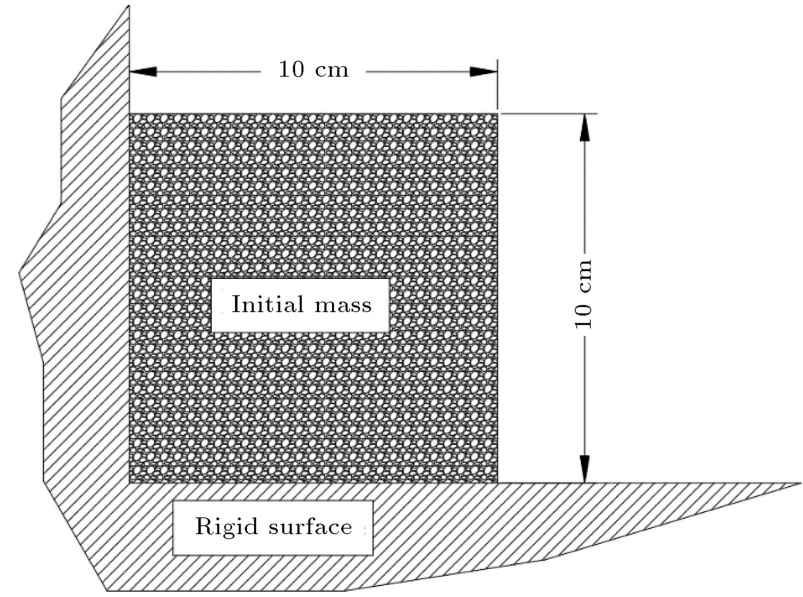

Figure 14. Schematic of a granular material column on a rigid surface.

In the present study, it is assumed that the variations in the bulk density of the granular material are negligible during flow and the rigid surface exhibits partial-slip conditions [33].

One of the most important indicators of collapses is the distance traveled by the moving material, called "runout", shown here by $X_{\infty}$. In the current numerical simulation, the slip length was changed to obtain runout values close to the experiments. A value $l_{s}=$ 0.016 for the slip length gave the best match with the experimental data.

The shape of the collapsed material is compared with the experimental results at different times in Figure 15. The assumption that the material bulk density remains constant during the collapse can be a reason why the results do not completely match. As can be seen from the results, by applying the rheological model, numerical modeling predicted the runout close to experiments at each elapsed time.

\subsubsection{Wet granular material}

In this section, the collapse of a two-dimensional trapezoidal column of granular material is investigated for different saturations. The aim is to study the effect of saturation on the final shape and runout of the collapsed material. Figure 16 shows a schematic of the problem.

The material properties are given in Table 1 . The slip length is set to $l_{s}=0.016 \mathrm{~m}$ and the bulk density of the wet material is obtained as follows:

$$
\rho_{\text {bulk }}=(1-\kappa) \rho+\kappa S \rho_{\text {Water }},
$$

where $\kappa$ is the porosity of material.

The final shapes of the collapsed material are compared in Figure 17 for different saturations. Figure 17 shows that:

$$
\begin{aligned}
\left(X_{\infty}\right)_{S=30 \%} & <\left(X_{\infty}\right)_{S=20 \%}<\left(X_{\infty}\right)_{S=10 \%} \\
& <\left(X_{\infty}\right)_{D r y}<\left(X_{\infty}\right)_{S \geq S_{\text {crit }}} .
\end{aligned}
$$




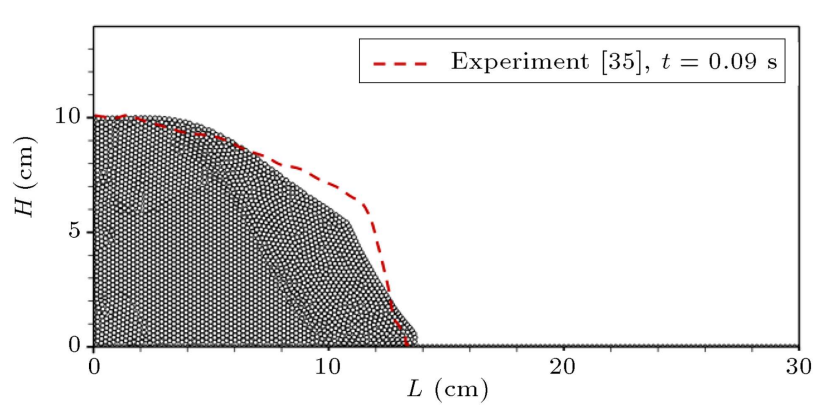

(a)

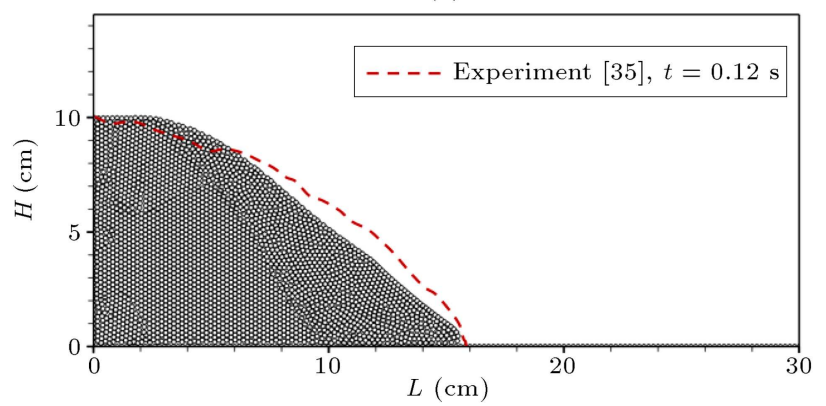

(b)

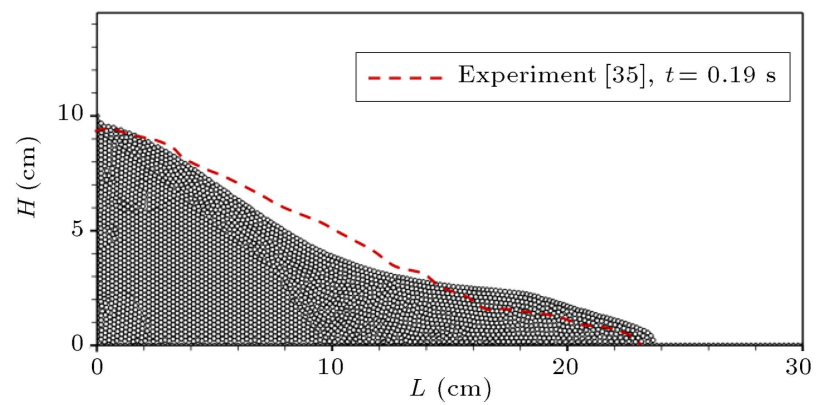

(c)

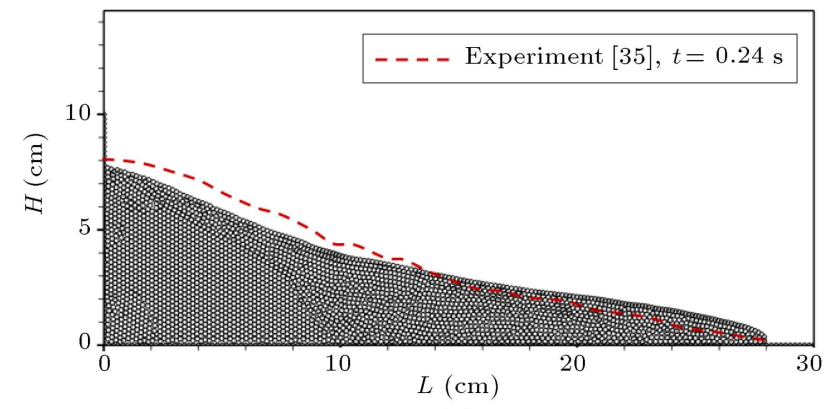

(d)

Figure 15. Comparison of free surface obtained by numerical simulation with experimental results [35] at different simulation times: (a) $t=0.09 \mathrm{~s}$, (b) $t=0.12 \mathrm{~s}$, (c) $t=0.19 \mathrm{~s}$, and (d) $t=0.24 \mathrm{~s}$.

Similar behavior can be observed in the amount of collapsed material (or collapsed area $\left(A_{\text {collapsed }}\right)$ ):

$$
\begin{aligned}
& \left(A_{\text {collapsed }}\right)_{S=30 \%}<\left(A_{\text {collapsed }}\right)_{S=20 \%} \\
& <\left(A_{\text {collapsed }}\right)_{S=10 \%}<\left(A_{\text {collapsed }}\right)_{D r y} \\
& <\left(A_{\text {collapsed }}\right)_{S} \geq S_{\text {crit }} .
\end{aligned}
$$

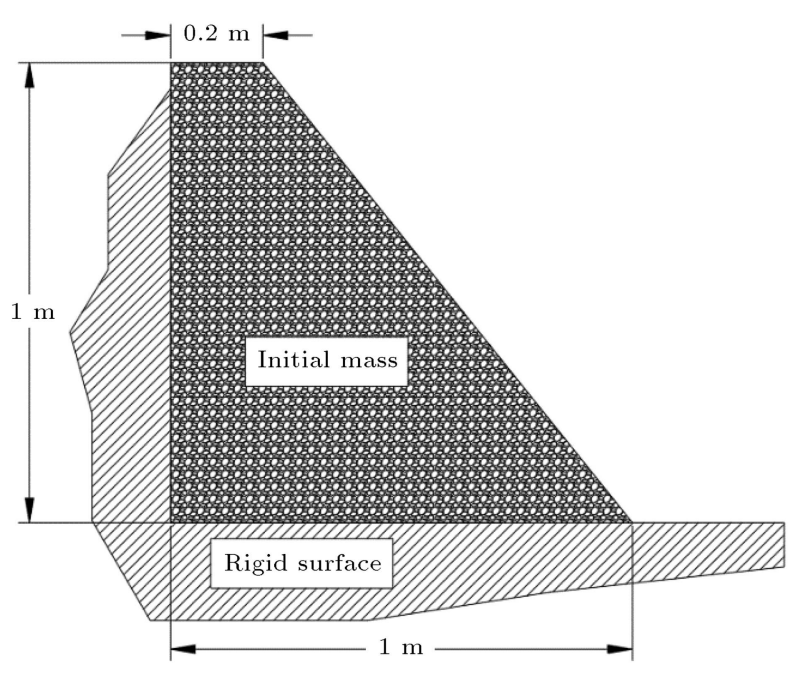

Figure 16. Schematic of trapezoidal column of granular material on a rigid surface.

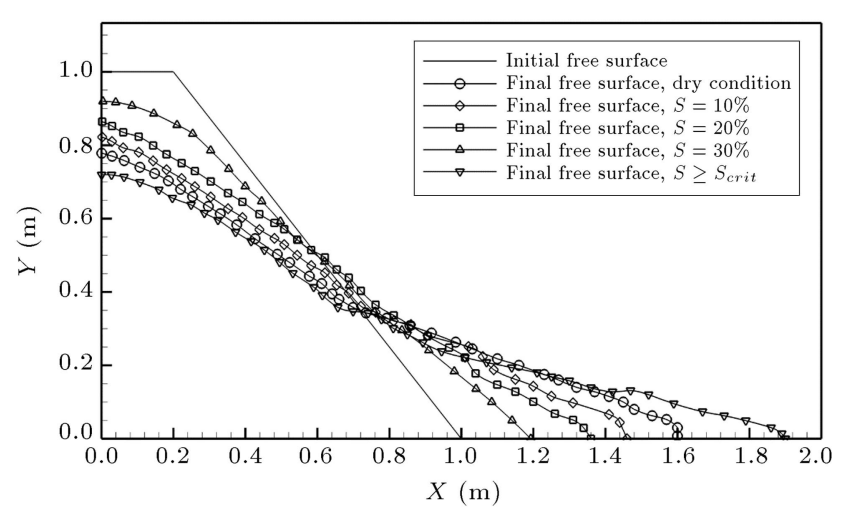

Figure 17. Final shapes of the collapsed material on a rigid surface for different saturations.

The reason is that by increasing saturation up to $S=S_{\text {crit }}$, the surface tension attractive force increases and the tendency of the material to flow decreases. However, when the saturation exceeds the critical value, the inter-particle water-induced attraction vanishes and due to buoyancy, the material becomes looser and the runout increases.

\section{Conclusion}

In this paper, a 3D DEM-based shear cell rheometer was used to obtain the rheological behavior of wet granular material. The granular material was considered as a homogenous and isotropic material composed of the same size particles. The main results of the present study are:

- During rainfall infiltration in a granular material, two main zones can be distinguished. The near surface saturated (and semi-saturated) zone with $S \geq S_{\text {crit }}$, lying above the unsaturated zone where $S<S_{\text {crit }}$. The critical saturation, $S_{\text {crit }}$, is the 
condition that the sign of pore water pressure changes from negative to positive;

- For the layers with $S \geq S_{\text {crit }}$, the pore pressure is hydrostatic and positive which increases with depth. In these layers, particles are fully wet and the buoyancy force reduces the effective contact stress between particles. Therefore, the material has lower apparent viscosity than dry material;

- For the layers with $0<S<S_{\text {crit }}$, the pressure in the inter-particle water is negative and the water surface tension pulls the particles towards each other. Therefore, the contact stress between particles increases, which in turn increases the shearing resistance. As a result, the material has higher apparent viscosity than the dry state;

- The rheological model of a granular material is obtained as a power-law equation and a function of inertia number for different saturations;

- The obtained rheological model predicted the changes in the final shape and the runout of the dry granular material close to the experiments in each elapsed time during the collapse;

- It is observed that by increasing saturation up to $S=S_{\text {crit }}$, the runout decreases. However, the runout of material with $S \geq S_{\text {crit }}$ is higher than the dry material.

\section{Nomenclature}

$\dot{\gamma}$

\section{Shear stress}

Shear rate

Yield stress

Apparent viscosity

Inertia number

Diameter

Density

Normal stress

Pore water pressure

Effective stress

Angle of internal friction

Particle radius

Half filling angle

Water - particle contact angle

Water bridge volume

Meniscus curvature radius

Saturation degree

Buoyancy force

Capillary force

Pressure difference between water bridge and air

\begin{tabular}{|c|c|}
\hline$\psi_{W A}$ & Water-air interfacial tension \\
\hline$A_{W e t}$ & Projection of wetted area in particle \\
\hline$F_{S}$ & Surface tension force \\
\hline$\delta_{\text {crit }}$ & Critical (rupture) distance \\
\hline$g$ & Gravitational acceleration \\
\hline$m$ & Mass \\
\hline$\vec{x}$ & Position vector \\
\hline$\omega$ & Angular velocity \\
\hline$F_{c}$ & Collision-induced force \\
\hline$\vec{d}$ & Distance vector \\
\hline$c$ & Contact point of two particle \\
\hline$\vec{n}$ & Normal unit vector \\
\hline$\vec{t}$ & Tangent unit vector \\
\hline$\nu$ & Linear velocity \\
\hline$\delta$ & Overlap value \\
\hline$k$ & Stiffness coefficient \\
\hline$\beta$ & Damper coefficient \\
\hline$V$ & Velocity \\
\hline$\lambda$ & $\begin{array}{l}\text { Tangential displacement of the contact } \\
\text { point }\end{array}$ \\
\hline$t$ & Time \\
\hline$E$ & Young modulus \\
\hline$G$ & Shearing modulus \\
\hline$v$ & Poisson ratio \\
\hline$\varepsilon$ & Restitution coefficient \\
\hline$\mu_{C}$ & Coulomb friction coefficient \\
\hline$\kappa$ & Porosity \\
\hline$X_{\infty}$ & Runout \\
\hline$l_{s}$ & Slip length \\
\hline
\end{tabular}

\section{References}

1. Iverson, R. "Landslide triggering by rain infiltration", Water Resources Research, 36, pp. 1897-1910 (2000).

2. Iverson, R. and George, D. "Modelling landslide liquefaction, mobility bifurcation and the dynamics of the 2014 Oso disaster", Géotechnique, 66, pp. 175-187 (2016).

3. Coussot, P., Rheometry of Pastes, Suspensions, and Granular Materials, Wiley, Hoboken, N.J (2010).

4. Saramito, P. and Wachs, A. "Progress in numerical simulation of yield stress fluid flows", Rheologica Acta, 56, pp. 211-230 (2017).

5. De Blasio, F., Introduction to the Physics of Landslides, Springer (2011).

6. Midi GDR. "On dense granular flows", The European Physical Journal E, 14, pp. 341-365 (2004).

7. Fall, A., Ovarlez, G., Hautemayou, D., et al. "Dry granular flows: Rheological measurements of the $\mu(I)$ rheology", Journal of Rheology, 59, pp. 1065-1080 (2015). 
8. Jop, P., Forterre, Y., and Pouliquen, O. "A constitutive law for dense granular flows", Nature, 441, pp. 727-730 (2006)

9. Gesenhues, L., Camata, J., and Coutinho, A. "Simulation of a column collapse for dense granular flows", American Congress on Computational Methods in Engineering (2017).

10. Valette, R., Riber, S., Sardo, L., et al. "Sensitivity to the rheology and geometry of granular collapses by using the $\mu(I)$ rheology", Computers \& Fluids, 191, p. 104260 (2019).

11. Gesenhues, L., Camata, J., Côrtes, A., et al. "Finite element simulation of complex dense granular flows using a well-posed regularization of the $\mu(I)$-rheology", Computers \& Fluids, 188, pp. 102-113 (2019).

12. Cundall, P. and Strack, O. "Discussion: A discrete numerical model for granular assemblies", Géotechnique, 30, pp. 331-336 (1980).

13. Bhateja, A. and Khakhar, D. "Rheology of dense granular flows in two dimensions: Comparison of fully two-dimensional flows to unidirectional shear flow", Physical Review Fluids, 3, p. 062301 (2018).

14. Mandal, S. and Khakhar, D. "A study of the rheology and micro-structure of dumbbells in shear geometries", Physics of Fluids, 30, p. 013303 (2018).

15. Shi, H., Roy, S., Weinhart, T., et al. "Steady state rheology of homogeneous and inhomogeneous cohesive granular materials", Granular Matter, 22, p. 14 (2020).

16. Binaree, T.H., Azéma, E., Estrada, N., et al. "Combined effects of contact friction and particle shape on strength properties and microstructure of sheared granular media", Physical Review E., 102, p. 022901 (2020).

17. Vo, T.T. and Nguyen-Thoi, T. "The role of interparticle friction on rheology and texture of wet granular flows", The European Physical Journal E., 43, p. 65 (2020).

18. Scheel, M., Seemann, R., Brinkmann, M., et al. "Morphological clues to wet granular pile stability", Nature Materials, 7, pp. 189-193 (2008).

19. Willett, C., Adams, M., Johnson, S., et al. "Capillary bridges between two spherical bodies", Langmuir, 16, pp. 9396-9405 (2000).

20. Zhao, C., Kruyt, N., and Millet, O. "Capillary bridge force between non-perfectly wettable spherical particles: an analytical theory for the pendular regime", Powder Technology, 339, pp. 827-837 (2018).

21. Kruyt, N. and Millet, O. "An analytical theory for the capillary bridge force between spheres", Journal of Fluid Mechanics, 812, pp. 129-151 (2017).

22. Nguyen, H., Millet, O., and Gagneux, G. "On the capillary bridge between spherical particles of unequal size: analytical and experimental approaches", Continuum Mechanics and Thermodynamics, 31, pp. 225-237 (2019).
23. Zhao, C., Kruyt, N., and Millet, O. "Capillary bridges between unequal-sized spherical particles: Rupture distances and capillary forces", Powder Technology, 346, pp. 462-476 (2019).

24. Schwarze, R., Gladkyy, A., Uhlig, F., et al. "Rheology of weakly wetted granular materials: a comparison of experimental and numerical data", Granular Matter, 15, pp. 455-465 (2013).

25. Obermayr, M., Vrettos, C., Eberhard, P., et al. "A discrete element model and its experimental validation for the prediction of draft forces in cohesive soil", Journal of Terramechanics, 53, pp. 93-104 (2014).

26. Dullien, F., El-Sayed, M., and Batra, V. "Rate of capillary rise in porous media with nonuniform pores", Journal of Colloid and Interface Science, 60, pp. 497506 (1977).

27. Urso, M., Lawrence, C., and Adams, M. "Pendular, funicular, and capillary bridges: results for two dimensions", Journal of Colloid and Interface Science, 220, pp. 42-56 (1999).

28. Seville, J. and Wu, C., Particle Technology and Engineering, Elsevier (2016).

29. Kloss, C., Goniva, C., Hager, A., et al. "Models, algorithms and validation for opensource DEM and CFDDEM", Progress in Computational Fluid Dynamics, An International Journal, 12, p. 140 (2012).

30. Shojaaee, Z., Roux, J., Chevoir, F., et al. "Shear flow of dense granular materials near smooth walls. I. Shear localization and constitutive laws in the boundary region", Physical Review E., 86, p. 011301 (2012).

31. da Cruz, F., Emam, S., Prochnow, M., et al. "Rheophysics of dense granular materials: Discrete simulation of plane shear flows", Physical Review E., 72, p. 021309 (2005).

32. Iverson, R., Reid, M., Logan, M., et al. "Positive feedback and momentum growth during debris-flow entrainment of wet bed sediment", Nature Geoscience, 4, pp. 116-121 (2010).

33. Nikooei, M. and Manzari, M. "Studying effect of entrainment on dynamics of debris flows using numerical simulation", Computers \& Geosciences, 134, p. 104337 (2020).

34. Hashemi, M., Fatehi, R., and Manzari, M. "A modified SPH method for simulating motion of rigid bodies in Newtonian fluid flows", International Journal of NonLinear Mechanics, 47, pp. 626-638 (2012).

35. Nguyen, C., Bui, H., and Fukagawa, R. "Failure mechanism of true 2D granular flows", Journal of Chemical Engineering of Japan, 48, pp. 395-402 (2015).

\section{Biographies}

Ramin Ghorbani received his BS (2012) and MS degrees (2014) in Mechanical Engineering with a minor in Energy Conversion. He is currently a PhD candidate at Sharif University of Technology. His researches are 
focused on the numerical modeling and simulation of granular flows.

Mehrdad T. Manzari received his $\mathrm{PhD}$ degree from the University of Wales Swansea in 1996. He has been a Professor of Mechanical Engineering at Sharif University of Technology since 2010. He was the Director of Thermo-Fluid Group (2007-2009), Director of Center of Excellence in Energy Conversion (20072009), and Director of Graduate Studies (2005-2007) at the School of Mechanical Engineering of Sharif University of Technology. His research interests are multiphase flows in porous media with applications in hydrocarbon reservoirs, simulation of fluid flow and heat transfer in manufacturing processes, fluid flow and heat transfer in micro-electro-mechanical-systems, non-Newtonian fluid flows, computational methods in engineering, and smoothed particle hydrodynamics with engineering applications.

Ali Hajilouy-Benisi received his $\mathrm{PhD}$ degree at the Mechanical Engineering Department of Imperial College at the University of London in 1993. He was a faculty member of the Institute of Water and Energy and since 1978, has been the faculty member at the School of Mechanical Engineering at Sharif University of Technology (SUT). He served as the Director of the Fluid Mechanics Lab (1993-1995), Founder and Director of the Turbocharger Lab (1993), Turbocharging Lab (2000), and Gas Turbine Lab (2008) at the School of Mechanical Engineering of Sharif University of Technology, Tehran, Iran. His research interests are experimental and theoretical investigations of turbochargers, turbocharging, and gas turbines. 